\title{
Consequences of mitotic slippage for antimicrotubule drug therapy
}

\author{
Bing Cheng' and Karen Crasta ${ }^{1,2,3,4}$ \\ ${ }^{1}$ Lee Kong Chian School of Medicine, Nanyang Technological University, Singapore, Singapore \\ ${ }^{2} \mathrm{School}$ of Biological Sciences, Nanyang Technological University, Singapore, Singapore \\ ${ }^{3} \mathrm{~A} *$ STAR Institute of Molecular and Cell Biology, Singapore, Singapore \\ ${ }^{4}$ Department of Medicine, Imperial College London, London, UK
}

Correspondence should be addressed to $\mathrm{K}$ Crasta

Email

kccrasta@ntu.edu.sg

\begin{abstract}
Antimicrotubule agents are commonly utilised as front-line therapies against several malignancies, either by themselves or as combination therapies. Cell-based studies have pinpointed the anti-proliferative basis of action to be a consequence of perturbation of microtubule dynamics leading to sustained activation of the spindle assembly checkpoint, prolonged mitotic arrest and mitotic cell death. However, depending on the biological context and cell type, cells may take an alternative route besides mitotic cell death via a process known as mitotic slippage. Here, mitotically arrested cells 'slip' to the next interphase without undergoing proper chromosome segregation and cytokinesis. These post-slippage cells in turn have two main cell fates, either cell death or a G1 arrest ensuing in senescence. In this review, we take a look at the factors determining mitotic cell death vs mitotic slippage, post-slippage cell fates and accompanying features, and their consequences for antimicrotubule drug treatment outcomes.
\end{abstract}

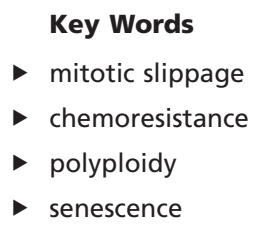

Endocrine-Related Cancer (2017) 24, T97-T106

\section{Introduction}

Antimitotic therapies are chemotherapeutic agents that target cells in mitosis. Of these, the antimicrotubule drugs (also known as microtubule poisons, spindle poisons or microtubule-targeting drugs) have proven to be the most successful in the clinics. These are commonlyadministered as first-line treatment against a variety of malignancies. These include ovarian, breast, head and neck, lung and prostate cancers, as well as various leukaemias (Jordan \& Wilson 2004). Microtubule poisons are divided into taxanes and vinca alkaloids depending on how they affect microtubule dynamics. Taxanes are microtubule-stabilising drugs that inhibit the dynamic instability of spindles; examples include commonly-used drugs such as paclitaxel and docetaxel. Vinca alkaloids depolymerise microtubules thereby preventing their attachment to kinetochores; drugs such as vinblastine, vincristine and vinolrelbine fall into this category (Allan \& Clarke 2007).

Accurate spindle function is crucial for a successful mitosis. A plethora of studies using cell culture systems have shown that perturbation of microtubule dynamics leads to sustained activation of the spindle assembly checkpoint (SAC) (Rieder \& Maiato 2004). The SAC prevents improper chromosome segregation and mitotic exit via inhibition of the anaphase-promoting complex (APC). Among its many roles, the APC has been shown to be responsible for controlling cyclin $\mathrm{B} 1$ levels during mitosis since APC-induced degradation of cyclin B1 engenders cells to exit mitosis (Lara-Gonzalez et al. 2012, Musacchio \& Ciliberto 2012). Thus, inhibiting the APC leads to cells arresting in mitosis. Depending on the biological context and cell type, cells blocked in mitosis can eventually

This paper forms part of a special section on 50 Years of Tubulin. The Guest Editors for this section were Karen Crasta and Ritu Aneja. 
undergo mitotic cell death, thereby fulfilling the antiproliferative function of antimicrotubule drugs (Rieder \& Maiato 2004).

Besides mitotic cell death, an alternative route that cells can also take following a SAC-enforced mitotic arrest is mitotic slippage. Attenuation of the SAC resulting in slow degradation of cyclin B1during a protracted mitotic arrest can cause cells to prematurely exit mitosis (Brito \& Rieder 2006). As illustrated in Fig. 1, cells 'slip' from mitosis and enter interphase without undergoing proper chromosome segregation and cytokinesis, yielding tetraploid multinucleated cells. Cells are then faced with three possible fates: (i) arrest in the G1 cell cycle phase; (ii) post-slippage cell death; or (iii) continue cycling as genomically unstable cells.

In the next few sections, it will become apparent that the tendency to 'slip' and the resultant post-slippage cell fate response vary significantly among cell lines treated with antimicrotubule drugs (Gascoigne \& Taylor 2008, Huang et al. 2010). Although antimicrotubule drugs have proven efficacious in the clinics, their long-term utility continues to be hampered by acquired chemoresistance and disease relapse. Most studies have focused on tubulin mutations and drug efflux pumps in a bid to understand mechanisms underlying drug resistance. Unfortunately, these findings have not been successfully translated to the clinics.

Here, we will discuss mechanisms by which mitotic slippage might potentially influence treatment outcomes and confer acquired resistance following antimicrotubule drug treatment. We start by describing roles of wellestablished factors in determining cell fate decision between mitotic cell death and mitotic slippage. This will then be followed by emerging evidence for the role of post-slippage cells in dictating outcomes and drug response to antimicrotubule drug therapy. Finally, we take a look at features associated with post-slippage cells that enter senescence.

\section{Factors that influence mitosis cell death vs mitotic slippage}

High-throughput time-lapse microscopy of single cells to address cell fate between mitotic cell death vs mitotic slippage revealed heterogeneity and stochasticity in signalling pathways activated by antimicrotubule drugs (Gascoigne \& Taylor 2008, Shi et al. 2008, Huang et al. 2010). This was observed not only among different cancer cell lines but also within different sub-populations within the same cell line as well. Since a high-degree of intra-line variation was apparent, Gascoigne and Taylor challenged the existing notion that cell fate was genetically predetermined. To do this, they carried out a pedigree analysis using chromosomally stable colon cancer cells HCT-116 (Gascoigne \& Taylor 2008). Daughter cells derived from the same mother were treated with the antimitotic drug AZ138 and subsequently tracked through the next mitosis. Their findings revealed that even genetically-identical daughter cells exhibited different cell fates in the next mitosis. In addition, they also analysed two non-transformed cell lines (human mammary epithelial HME cells and retinal pigment epithelial RPE-1 cells) that lacked genomic instability and were therefore considered genetically homogeneous. Both these cell

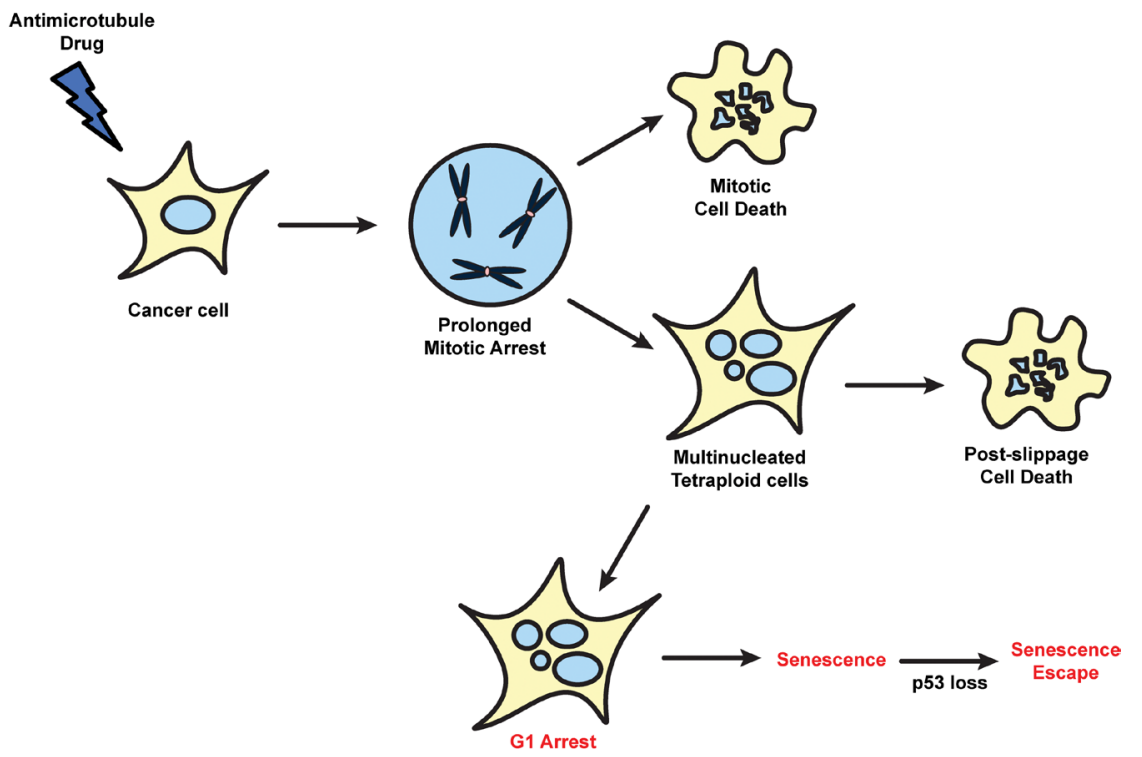

Figure 1

Different cell fates following antimicrotubule drug treatment. Cancer cells treated with antimicrotubule drugs go through a prolonged mitotic arrest that can culminate in mitotic cell death. Mitotically arrested cells can also take an alternative route known as mitotic slippage and escape mitotic cell death. Here, cells exit mitosis prematurely, without proper chromosome segregation and cytokinesis, and enter the next interphase as multinucleated tetraploid cells. The main post-slippage cell fates are shown: cells either undergo a G1 arrest, often leading to senescence or post-slippage cell death. 
lines also exhibited multiple different cell fates. These results supported the conclusion that cell fates following a mitotic arrest are not genetically predetermined.

It had long been assumed that chromosomally unstable cells possess a weakened SAC. However, cell fate profiling of chromosomal instability (CIN cell lines) and microsatellite instability (MIN) cell lines by live-cell imaging revealed that this was not the case (Gascoigne \& Taylor 2008). Time-lapse microscopy analyses revealed that CIN cells arrested in mitosis for similar duration or at times even longer than chromosomally stable MIN cells. This proved that the SAC was functional in CIN cells following antimicrotubule drug treatment. Hence, the notion that CIN cells are more sensitive to antimicrotubule drugs was not supported.

The 'competing networks-threshold' model best explains the tendency of a cell to either die in mitosis or to undergo mitotic slippage following SAC activation and mitotic arrest. The model posits that cell fate following mitotic arrest is dictated by two independent networks, namely pro-apoptotic caspase activation and cyclin B1 degradation (Gascoigne \& Taylor 2008). Both networks contain thresholds and cell fate is determined by which threshold is breached first. If cyclin B1 levels fall below the mitotic exit threshold before caspase activation, mitotic slippage occurs. Conversely, if caspase activation occurs before cyclin B1 is sufficiently degraded and the threshold for apoptosis is breached, mitotic cell death occurs.

In support of this, a genome-wide siRNA screen in Taxol-treated HeLa cells led to the finding that p31 comet (inhibitor of SAC component Mad2) promoted mitotic slippage via cyclin B1 degradation, whereas proapoptotic factor Noxa initiated mitotic cell death (DiazMartinez et al. 2014). These findings also revealed possible crosstalk between competing mitotic cell death and mitotic slippage pathways, suggesting an additional mechanism besides the independent 'competing networks-threshold' model described above. This was through the activation of the mitochondrial apoptotic pathway which was shown to play a role in modulating mitotic slippage. Both depletion of Bax and Bak (pro-apoptotic factors), and that of DRP1 (mitochondrial fission factor) lengthened mitotic duration and delayed mitotic slippage in mitotic slippageprone U2OS cells (Diaz-Martinez et al. 2014).

Additionally, recent studies reported that the ubiquitin ligase CRL2ZYG-11 could promote mitotic slippage in SAC-arrested cells by targeting degradation of cyclin B1 (Balachandran et al. 2016). Depletion of the ZYG11A/B subunit in nocodazole-treated cells dramatically decreased the frequencies of post-slippage cells.
Interestingly, mitotic slippage was completely inhibited in the combined presence of a CRL ubiquitin ligase pan-inhibitor MLN4924 and nocodazole, leading to a hundred percent cell death during mitotic arrest. Hence, elucidating combination therapies with antimicrotubule drugs to block mitotic slippage could prove to be an efficacious means of ensuring the antimitotic cytotoxic function of these drugs.

Although mechanisms contributing to cyclin B1 regulation have been well-studied, the pro-apoptotic pathway is less well-understood. A recent study demonstrated that the dynamics of myeloid cell leukaemia-1 (Mcl-1), a pro-survival member of the Bcl-2 family, could influence cell fate decision between mitotic slippage and mitotic cell death (Sloss et al. 2016). Mc1-1 was shown to be continuously synthesised and degraded during mitosis. The depletion of Mcl-1 by siRNA transfection was found to accelerate mitotic cell death, suggesting that Mc1-1 degradation during mitotic arrest could serve as a 'mitotic death timer'. Mcl-1 levels were also found to modulate mitotic slippage. Consistent with the pro-survival function of Mcl-1, the overexpression of Mc1-1 in Taxol-treated cells was found to prolong mitosis and delay mitotic slippage. Conversely, Mcl-1 inhibition in mitotic slippage-prone cells accelerated slippage and post-slippage death. This was suggested to be due to the ability of Mc1-1 to compete with cyclin B1 for the proteolytic machinery, which led to cyclin B1 attenuation and consequently, slippage.

The depletion of Bcl-xL by siRNA transfection was also found to accelerate mitotic cell death (Sloss et al. 2016). Indeed, work from the Mitchison group showed that both Mcl-1 and Bcl-xL serve as primary negative regulators of apoptosis during a prolonged mitotic arrest (Shi et al. 2011). In addition, the Bcl-2 family inhibitor navitoclax was also found to accelerate mitotic cell death by antagonising Bcl-xL (Shi et al. 2011). The importance of Bcl-xL for mitotic cell death was further confirmed by experiments where BH3-mimetic-mediated inhibition of Bcl-xL sensitised cells to microtubule-binding agents, kinesin inhibitors and mitotic kinase inhibitors (Bennett et al. 2016). It was also shown that Bcl-xL inhibition only had a minor effect when used with inhibitors that overrode the SAC causing slippage (Bennett et al. 2016).

The level of cyclin-dependent kinase1 (Cdk1) has been postulated to control both cell death and mitotic exit. Studies have shown that phosphorylation of the apoptotic initiator protease caspase- 9 at Thr125 by Cdk1/cyclin B1 reduced its activity, thus protecting mitotic cells from apoptosis (Allan \& Clarke 2007). In addition,

Published by Bioscientifica Ltd. 
phosphorylation of Mcl-1 at Thr92 by Cdk1/cyclin B1 caused its APC-mediated degradation, thus triggering apoptosis during mitotic arrest (Harley et al. 2010). Indeed, a mitotic death signature using several cell lines has been identified based on the hypothesis that mitotic cell death occurs as a result of robust Cdk1 signalling where Mcl-1 and Bcl-xL are completely phosphorylated and thereby degraded or inactivated (Sakurikar et al. 2012, 2014). Conversely, mitotic slippage was associated with incomplete phosphorylation of Mcl-1 and Bcl-xL. However, presence of such a signature in tumour cells remains to be validated and warrants further testing in a clinical setting.

For further discussion on the cell death response during mitotic arrest, the reader is referred to the comprehensive review by Shi and Mitchison in this issue.

In the following sections, we will discuss cell response to antimicrotubule drug treatment exclusively after mitotic slippage.

\section{Post-slippage cell death and cytotoxic drug response}

Mitotically arrested cells have been shown to adopt different cell fates following mitotic slippage. These include death, G1 cell cycle arrest followed by senescence, or cells can even exist as viable polyploid cells capable of re-entering the cell cycle leading to chromosomal instability (CIN) (Weaver \& Cleveland 2005).

Cell death during interphase is another means by which the cytotoxic goal of antimicrotubule treatment can be achieved. DNA damage and strong p53 induction had been observed following mitotic slippage (Orth et al. 2012). This was attributed to partial activation of caspase activity during a prolonged mitotic arrest. This rendered DNA fragmentation and a p53-mediated DNA damage response in post-slippage cells (Quignon et al. 2007, Orth et al. 2012). Work from the Shi Lab further showed that the DNA damage response pathway was primarily responsible for post-slippage apoptosis in a p53-dependent manner (Zhu et al. 2014). In support of this, DNA damage inhibition and knockdown of p53 by siRNA transfection both reduced post-slippage cell death following paclitaxel treatment. In addition, post-slippage multinucleation was suggested to contribute to post-slippage cell death since attenuation of the extent of multinucleation dramatically reduced the incidence of DNA damage and apoptosis (Zhu et al. 2014).

A recent study which utilised chemical inhibitors against centromere-associated protein CENP-E and kinesin-related protein Eg5 to study cell fates following mitotic slippage may have potential implications for antimicrotubule drug treatment (Ohashi et al. 2015). CENP-E is a kinesin-like motor protein that controls chromosome alignment, whereas Eg5 is a motor protein that regulates centrosome separation and bipolar spindle formation. Under SAC-impaired conditions, inhibition of CENP-E led to aneuploidy through chromosome mis-segregation after mitotic slippage, while that of Eg5 led to polyploidy through cytokinesis failure due to monopolar spindle formation. Importantly, the study revealed that although both conditions allowed escape from cell death during mitotic arrest, aneuploidy, but not polyploidy, led to post-slippage apoptosis (Ohashi et al. 2015). This post-slippage apoptosis was shown to be p53-mediated and accompanied by aneuploidy-activated DNA damage response and proteotoxic stress. Hence, CENP-E inhibitors could potentially be used in SAC-impaired tumours resistant to current antimicrotubule drugs.

\section{Checkpoints, mitotic slippage and drug resistance}

Reports describing molecular mechanisms underlying antimicrotubule drug resistance have mainly focused on the cellular effects of various SAC components. In particular, a nexus between a weakened SAC and antimicrotubule drug resistance in vitro has become apparent (Weaver \& Cleveland 2005, Yamada \& Gorbsky 2006). The first evidence of paclitaxel sensitivity being dependent on a functional SAC demonstrated that downregulation of SAC components Mad2 and BubR1 increased cell survival (Sudo et al. 2004). Since then, a few studies have demonstrated a correlation between increased incidence of mitotic slippage and drug resistance in vitro. For instance, cells overexpressing p31 comet displayed increased tendencies to enter mitotic slippage and cell survival following antimicrotubule drug treatment in cancer cell lines (Ma et al. 2012, Habu \& Matsumoto 2013). In addition, paclitaxel-resistant ovarian cancer cell line showed weakened SAC with reduced BubR1 expression (Fu et al. 2007). In addition, inhibition of mitotic slippage by cell cycle regulator Cdc6 depletion was proposed to decrease cell survival (He et al. 2016).

Although a role for mitotic slippage in drug resistance has been proposed in vitro, post-slippage cellular fates and their associated mechanisms contributing to drug response have not been addressed in these studies. In the next few sections, we discuss a few features and outcomes

Published by Bioscientifica Ltd. 
associated with mitotic slippage that may account for this potential role in drug resistance.

\section{Effects of cellular senescence and SASP in cancer therapy}

Besides post-slippage apoptosis, another cellular fate that has been described is cellular senescence (Rieder \& Maiato 2004). Cellular senescence occurs as a consequence of sustained stress which activates a response governed by the p53 and pRB tumour suppressor proteins (Campisi \& d'Adda di Fagagna 2007). This triggers a signalling pathway that enforces a stable cell cycle arrest.

Cellular senescence has been shown to limit tumourigenesis as well as foster anti-proliferative response to cancer therapy. Sustained expression of oncogenic BRAFV600E mutation in melanocytes was found to rarely progress to malignancy (melanomas). Following increased proliferation and growth, these melanocytes induce senescence, displaying SA- $\beta$-gal-positive cells and p16 expression (Michaloglou etal. 2005). Hence, the senescence phenotype was largely attributed to suppressing malignant transformation in benign melanocytic nevi. Indeed, the senescence phenotype has also been observed in other benign tumour types such as lung adenomas but not in adenocarcinomas, and in benign prostatic hyperplasia (Choi et al. 2000, Majumder et al. 2008). Additionally, inactivation of senescence pathways was reported to be necessary for progression to malignancy. For example, the dual inactivation of p16 and p53 led to developmental of pancreatic cystic neoplasm (Bardeesy et al. 2002). Interestingly, it was shown that although cells carrying defective tumour suppressor PTEN still undergo senescence, the inactivation of p53 was sufficient to drive cells towards tumourigenesis (Chen et al. 2005). Conversely, reactivation of functional p53 in mice tumour models led to induction of senescence and tumour regression (Ventura et al. 2007, Xue et al. 2007), illustrating that senescence can indeed serve as a natural barrier and limit tumour growth.

Due to the role of senescence as a tumour suppressor, pro-senescence therapies have been proposed as a potential cancer therapy strategy (Nardella et al. 2011, Acosta \& Gil 2012). For instance, cellular senescence has been observed in cells as well as in lung cancer patients receiving neo-adjuvant chemotherapy (Roberson et al. 2005). The carboplatin/paclitaxel post-treatment tumour biopsies from patients with non-small cell lung cancer, but not from untreated patients, displayed SA- $\beta$-gal staining. Senescence was also correlated with tumour regression.
Upregulated Cdc2/Cdk1 was associated with senescence bypass, both in cell-based studies in vitro as well as in patient samples (Roberson et al. 2005). Inhibitors of Cdk1 was thus proposed to be used as combinatorial treatment with conventional chemotherapy.

Senescent cells are metabolically active and exhibit a complex senescent cell secretome, a feature referred to as the senescence-associated secretory phenotype (SASP) (Kuilman \& Peeper 2009, Coppe et al. 2010). The SASP consists of a wide range of factors consisting of inflammatory cytokines, chemokines, metalloproteases and extracellular matrices that can alter the tumour microenvironment. In addition to an intrinsic cellautonomous tumour-suppressive mechanism, work from the Gil and Peeper groups showed that SASP components such as IL- 6 and IL-8 reinforced senescence in an autocrine manner (Acosta et al. 2008, Kuilman et al. 2008). A more recent study from the Gil lab has also shown that SASP components can also induce paracrine pro-senescence signalling (Acosta et al. 2013). The paracrine transmission of senescence from cells that have undergone oncogeneinduced senescence to primary cells was shown to be mediated by TGF- $\beta$ and interleukin IL-1. Inflammasomes were shown to mediate SASP signalling, demonstrating a link to innate immunity.

While SASP factors can exert tumour suppression by reinforcing senescence or inducing paracrine senescence (Acosta et al. 2008, 2013), these factors can also promote tumourigenesis, particularly via its paracrine inflammatory and growth-promoting properties (Coppe et al. 2008). For example, it has been shown that secreted factors from senescent fibroblasts could promote malignant transformation of premalignant epithelial cells, and stimulate tumour vascularisation (Krtolica et al. 2001, Bavik et al. 2006). Hence, the SASP can evoke a pro-tumourigenic microenvironment where SASP factors confer paracrine tumourigenic effects on neighbouring premalignant cells. Studies describing this non-cellautonomous function include epithelial-mesenchymal transition and invasion, tumour vascularisation, immune surveillance and abnormal tissue morphology, mediated by pro-inflammatory cytokines IL-6 and IL-8, metalloprotease MMP3 and VEGF (Parrinello et al. 2005, Coppe et al. 2006, 2008, Kuilman et al. 2008, Davalos et al. 2010).

Thus far, we have discussed a few examples of the biological impact of senescence and SASP in tumourigenesis. In the context of this review, we look at a few studies that have explored links between paclitaxel response and senescence in vitro. Cellular senescence

Published by Bioscientifica Ltd 
induced by Mad2 knockdown has been implicated in paclitaxel resistance (Prencipe et al. 2009). Here, cells with reduced Mad2 levels were found to undergo mitotic slippage-associated senescence and secreted cytokines IL-6 and IL-8. This senescence phenotype was enhanced by paclitaxel treatment. Cell viability assays of cells with compromised Mad2 treated with paclitaxel showed increased cell survival compared to the control, suggesting that senescent cells could evade paclitaxel-induced cell death (Prencipe et al. 2009). Similarly, Mad2 or BubR1depleted gastric cancer cell lines also displayed enhanced cellular senescence following paclitaxel treatment (Bargiela-Iparraguirre et al. 2014). Work done in our lab (B Cheng and $\mathrm{K}$ Crasta, unpublished observations) focused on mitotic slippage-prone cells that entered senescence following treatment with nocodazole and paclitaxel. This has provided some insights into acquired resistance following mitotic slippage (Fig. 2). We found that mitotic slippage-induced senescent cells elicited SASP both in vitro and in vivo. Post-slippage SASP factors, particularly the pro-inflammatory cytokines, conferred pro-tumourigenic effects on neighbouring cells, possibly offering an explanation for a mechanism of antimicrotubule drug resistance. Work is currently underway to elucidate underlying associated mechanisms.

Despite the importance of the SASP, little is known about the regulation behind its phenotypic effects. Indeed, insights into cell fate determination between tumour-suppressive vs pro-tumourigenic phenotypes remain scarce. The p38MAPK and the mammalian target of rapamycin (mTOR) pathway have clearly emerged as central players in controlling SASP factor production (Freund et al. 2011, Herranz et al. 2015, Laberge et al. 2015). Here, we focus on regulation by the mTOR pathway as it has been better characterised.
mTOR inhibition by rapamycin was shown to suppress the secretion of major SASP factors, including the pro-inflammatory cytokine IL-6 (Laberge et al. 2015). Interestingly, modulation of these SASP proteins was found to be at the mRNA level and not merely due to a decrease in protein synthesis. The transcription of SASP proteins is largely regulated by positive feedback loops between pro-inflammatory cytokines and the transcription factor NF- $\mathrm{kB}$, locally amplifying the pro-inflammatory cascade. For example, proinflammatory cytokines IL-6 and IL-8 were shown to confer pro-senescence effects in 'senescence-primed' cells via autocrine feedback loops (Acosta et al. 2008, Kuilman et al. 2008). Regulation of IL-6 and IL-8 in reinforcing senescence was shown to be controlled by IL- $1 \alpha$ signalling (Orjalo et al. 2009). In addition, conditioned medium from IL-1 $\alpha$-depleted senescent cells was observed to dramatically reduce the IL-6/IL-8dependent invasiveness of metastatic cancer cells. This suggests that IL- $1 \alpha$ regulates the biological effects of these cytokines. IL- $1 \alpha$ has been shown to be an upstream regulator of a pro-inflammatory network within the SASP through the activation of NF-кB (Acosta et al. 2008, Orjalo et al. 2009, Freund et al. 2011). Studies using rapamycin also suggested that mTOR facilitates IL- $1 \alpha$ translation, leading to NF-kB activation (Laberge et al. 2015). This triggered the amplification of the downstream pro-inflammatory cytokine network through positive feedback between NF-кB and cytokines including IL-1 $\alpha$. Another study from the Gil lab also showed mTOR inhibitor rapamycin to be a potent SASP suppressor (Herranz et al. 2015). Here, mTOR controlled the SASP by differentially regulating the translation of MAPKAPK2 kinase through the eukaryotic translation initiation factor 4EBP1. MAPKAPK2 was found to phosphorylate the RNA-binding protein ZFP36L1, inhibiting its ability

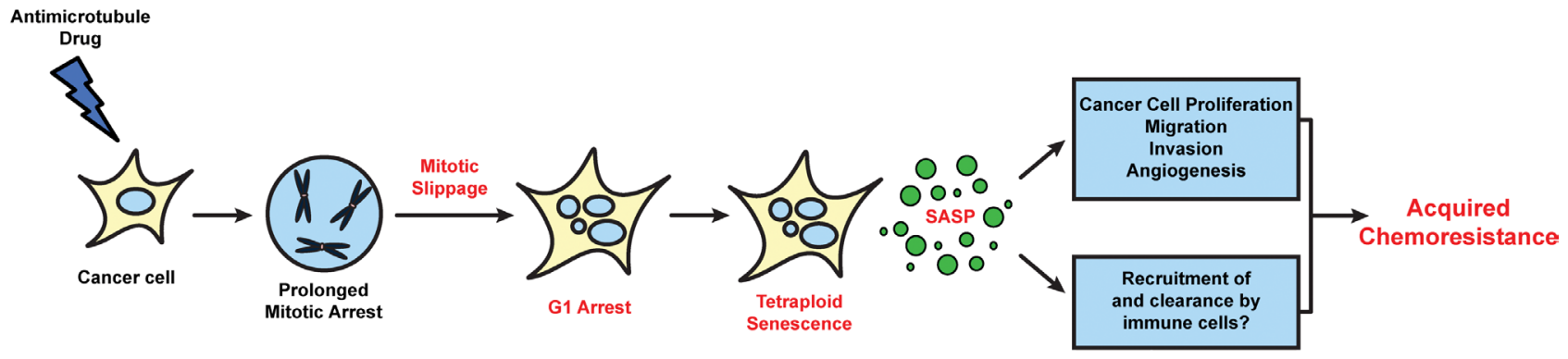

Figure 2

Different cell fates following antimicrotubule drug treatment. Cells can undergo mitotic slippage-induced senescence after antimicrotubule drug treatment. These cells are capable of eliciting the senescence-associated secretory phenotype (SASP) that confer paracrine pro-tumourigenic effects such as cell proliferation, migration, invasion and angiogenesis. This can potentially contribute to the emergence of acquired chemoresistance. 
to degrade the transcripts of several SASP components. Notably, mTOR inhibition and constitutive activation of ZFP36L1 impaired the paracrine effects of senescent cells in both tumour-suppressive and tumour-promoting contexts (Herranz et al. 2015).

The SASP can also promote tumour growth by establishing an immunosuppressive microenvironment (Toso et al. 2014, Ruhland et al 2016). It was previously shown that temporary and selective inactivation of tumour suppressor PTEN could induce hyperactivation of a signalling pathway that led to senescence (Alimonti et al. 2010). As a follow-up to this, work from the Alimonti lab showed that in PTEN-null senescent tumours, the activation of Jak2/Stat3 pathway established an immunosuppressive tumour microenvironment which contributed to tumour growth and chemoresistance (Toso et al. 2014). Significantly, combinatorial treatment with docetaxel and a JAK2 inhibitor reprogrammed the SASP. This was shown to enhance the drug response of docetaxel-induced senescent cells by creating a strong immunosuppressive condition in PTEN-null tumours (Toso et al. 2014). These studies demonstrate the importance of suppressing immune surveillance of senescent tumour cells engendered by drug treatments. On a related note, a recent report (Ruhland et al. 2016) showed that senescent stromal cells were sufficient to create an immunosuppressed environment. Here, stromal-derived SASP factor IL-6 recruited suppressive myeloid cells and inhibited anti-tumour T-cell response. This established a tumour-permissive environment unabated by the immune system. Hence, the inflammatory properties of the SASP pose ramifications for therapy-induced senescence where pro-tumourigenic effects of SASP factors may antagonise desired clinical outcome, possibly driving resistance to drug treatment.

\section{Polyploidy, multinucleation and chemoresistance}

We next examine other prominent attributes of postslippage cells. Polyploidy (an increase in the sets of chromosomes) and multinucleation are characteristic features of mitotic slippage. Tetraploidy, or successive polyploidizaton if these cells undergo successive cell cycles, was first described by early studies that sought to correlate polyploidy, micronuclei and cell survival following exposure to nocodazole (Elhajouji et al. 1998, Decordier et al. 2008).

In the tumourigenesis context, tetraploids are often found in pre-cancerous lesions and early stages of a variety of cancers (Vitale et al. 2011). Depending on the p53 status, these cells are capable of undergoing apoptosis spontaneously or reverting to aneuploid cells with tumourigenic potential (Vitale et al. 2011). Additionally, it was found that p53-null tetraploid mouse mammary epithelial cells MMEC, but not their diploid equivalent, led to increased chromosomal abnormalities and transformation (Fujiwara et al. 2005). Furthermore, tetraploid fibroblasts were shown to exhibit high frequencies of spontaneous DNA damage and decreased DNA repair, contributing to further genomic instability (Thorpe et al. 2007).

Polyploidisation has been described in early reports of paclitaxel-resistant human leukaemia cells in vitro (Roberts et al. 1990). Indeed, Docetaxel-induced polyploidisation has been proposed to underlie chemoresistance and disease relapse (Mittal et al. 2017). Additionally, the Kroemer lab described selective resistance of tetraploid cancer cells against DNA damage-induced apoptosis (Castedo et al. 2006). As described early in this review, aneuploidy, but not polyploidy, was found to cause post-slippage apoptosis (Ohashi et al. 2015). This raises the possibility that polyploid cells may possess potential proclivity towards post-slippage senescence rather than post-slippage apoptosis. Notably, polyploidisation has been correlated with cellular senescence (Mosieniak \& Sikora 2010). Polyploid cells have also been described to confer chemoresistance by undergoing senescence and subsequently re-entering the cell cycle (Wang et al. 2013). Cells that escape senescence have been reported to possess chemoresistant or cancer stem-like properties (Achuthan et al. 2011, Zhang et al. 2014).

Multinucleation is one of the overt morphological characteristics of post-slippage cells. Micronuclei formed from nocodazole-induced mis-segregated chromosomes have been shown to develop DNA breaks in the next interphase (Crasta et al. 2012). Following mitotic slippage, multinucleated cells have also been shown to harbour DNA damage (Zhu et al. 2014). Attenuation of the extent of multinucleation dramatically reduced the incidence of DNA damage and apoptosis, suggesting that multinucleation contributes to post-slippage cell death (Zhu et al. 2014). We propose that it is the degree of DNA damage in multinucleated cells that dictates cell survival or death following mitotic slippage. A lower extent of DNA damage in multinucleated cells may contribute to cellular senescence and drug resistance (B Cheng and $\mathrm{K}$ Crasta, unpublished observations). Indeed, multinucleation was observed to be an early step in drug resistance in an early study describing paclitaxel action

Published by Bioscientifica Ltd. 
(Panvichian et al. 1998). Extensive multinucleation has also been shown to correlate with cell survival and chemoresistance (Makarovskiy et al. 2002, Martin et al. 2016, Mittal et al. 2017).

\section{Concluding remarks and future perspectives}

The anti-proliferative basis for antimicrotubule cytotoxic killing has long thought to be due to the presence of an activated SAC that culminates in a mitotic cell death (Rieder \& Maiato 2004). However as discussed, depending on the biological context and cell type, cells can 'opt for' mitotic slippage instead of mitotic death.

In this review, we have seen various examples of mitotic slippage being a potential mechanism limiting the effectiveness of antimicrotubule drugs. For instance, MLN4924, which inhibits mitotic slippage in human cells, has recently been proposed as a beneficial combination therapy (Balachandran et al. 2016). Indeed, the Mitchison lab has shown mitotic exit to be a better cancer therapeutic target than the SAC (Huang et al. 2009). This also underscores the need for better understanding of the molecular mechanisms underlying the various cell fates following mitotic slippage. We have also discussed the potential of paclitaxel-induced senescence in conferring cell survival. However, it is important to bear in mind that the in vitro conditions reported may not fully recapitulate aspects of the tumour microenvironment to be translated effectively into improved patient outcomes. Hence, additional studies are needed to confirm these findings using tractable mouse models or clinical patient samples. It will also serve one well to bear in mind that genetic events and tissue contexts are important factors to consider in modulating apoptotic or senescent activities; TP53 is a prime example on how the cell fate decision can be altered in the presence of wildtype vs deficient p53. Additionally, further investigation into delineating how senescent cells affect the immune system and are removed by it during mitotic slippage-induced senescence is required. Another important area of study would be the identification of pathways that influence the development and function of SASP factors. Since the senescence state serves as an anticancer barrier, eliminating or targeting senescent cells may pose a risk instead of alleviating resistance. As such, a state of 'mitotic slippage-induced senescence without SASP' may be ideal. Modulating SASPmediated pathways without interfering with senescence will thus have potential to combat antimicrotubule drug resistance. We propose that combinatorial therapy with inhibitors that bypass or target post-slippage SASP may be beneficial towards improving patient outcomes following antimicrotubule drug treatment.

Declaration of interest

The authors declare that there is no conflict of interest that could be perceived as prejudicing the impartiality of this review.

\section{Funding}

This work was supported by the National Research Foundation (NRFNRFF2013-10) and the Nanyang Assistant Professorship Grant, Nanyang Technological University.

Author contribution statement

$\mathrm{K} C$ and $\mathrm{C} \mathrm{B}$ wrote the manuscript and $\mathrm{K} C$ supervised the study.

\section{References}

Achuthan S, Santhoshkumar TR, Prabhakar J, Nair SA \& Pillai MR 2011 Drug-induced senescence generates chemoresistant stemlike cells with low reactive oxygen species. Journal of Biological Chemistry 286 37813-37829. (doi:10.1074/jbc.M110.200675)

Acosta JC \& Gil J 2012 Senescence: a new weapon for cancer therapy. Trends Cell Biology 22 211-219. (doi:10.1016/j.tcb.2011.11.006)

Acosta JC, O’Loghlen A, Banito A, Guijarro MV, Augert A, Raguz S, Fumagalli M, Da Costa M, Brown C, Popov N, et al. 2008 Chemokine signaling via the CXCR2 receptor reinforces senescence. Cell 133 1006-1018. (doi:10.1016/j.cell.2008.03.038)

Acosta JC, Banito A, Wuestefeld T, Georgilis A, Janich P, Morton JP, Athineos D, Kang TW, Lasitschka F, Andrulis M, et al. 2013 A complex secretory program orchestrated by the inflammasome controls paracrine senescence. Nature Cell Biology 15 978-990. (doi:10.1038/ncb2784)

Alimonti A, Carracedo A, Clohessy JG, Trotman LC, Nardella C, Egla A, Salmena L, Sampieri K, Haveman WJ, Brogi E, et al. 2010 Subtle variations in Pten dose determine cancer susceptablity. Nature Genetics 42 454-458. (doi:10.1038/ng.556)

Allan LA \& Clarke PR 2007 Phosphorylation of caspase-9 by CDK1/cyclin B1 protects mitotic cells against apoptosis. Molecular Cell 26 301-310. (doi:10.1016/j.molcel.2007.03.019)

Balachandran RS, Heighington CS, Starostina NG, Anderson JW, Owen DL, Vasudevan S \& Kipreos ET 2016 The ubiquitin ligase CRL2ZYG11 targets cyclin B1 for degradation in a conserved pathway that facilitates mitotic slippage. Journal of Cell Biology 215 151-166. (doi:10.1083/jcb.201601083)

Bardeesy N, Morgan J, Sinha M, Signoretti S, Srivastava S, Loda M, Merlino G \& DePinho RA 2002 Obligate roles for p16(Ink4a) and p19(Arf)-p53 in the suppression of murine pancreatic neoplasia. Molecular and Cellular Biology 22 635-643. (doi:10.1128/ MCB.22.2.635-643.2002)

Bargiela-Iparraguirre J, Prado-Marchal L, Pajuelo-Lozano N, Jimenez B, Perona R \& Sanchez-Perez I 2014 Mad2 and BubR1 modulates tumourigenesis and paclitaxel response in MKN45 gastric cancer cells. Cell Cycle 13 3590-3601. (doi:10.4161/15384101.2014.962952)

Bavik C, Coleman I, Dean JP, Knudsen B, Plymate S \& Nelson PS 2006 The gene expression program of prostate fibroblast senescence modulates neoplastic epithelial cell proliferation through paracrine mechanisms. Cancer Research 66 794-802. (doi:10.1158/0008-5472.CAN-05-1716)
(C) 2017 Society for Endocrinology Printed in Great Britain
Published by Bioscientifica Ltd. 
Bennett A, Sloss O, Topham C, Nelson L, Tighe A \& Taylor SS 2016 Inhibition of Bcl-xL sensitizes cells to mitotic blockers, but not mitotic drivers. Open Biology 6 160134. (doi:10.1098/rsob.160134)

Brito DA \& Rieder CL 2006 Mitotic checkpoint slippage in humans occurs via cyclin B destruction in the presence of an active checkpoint. Current Biology 16 1194-1200. (doi:10.1016/j. cub.2006.04.043)

Campisi J \& di Fagagna d'Adda F 2007 Cellular senescence: when bad things happen to good cells. Nature Reviews Molecular Cell Biology $\mathbf{8}$ 729-740. (doi:10.1038/nrm2233)

Castedo M, Coquelle A, Vitale I, Vivet S, Mouhamad S, Viaud S, Zitvogel L \& Kroemer G 2006 Selective resistance of tetraploid cancer cells against DNA damage-induced apoptosis. Annals of the New York Academy of Sciences 1090 35-49. (doi:10.1196/annals.1378.004)

Chen Z, Trotman LC, Shaffer D, Lin HK, Dotan ZA, Niki M, Koutcher JA, Scher HI, Ludwig T, Gerald W, et al. 2005 Crucial role of p53dependent cellular senescence in suppression of Pten-deficient tumorigenesis. Nature 436 725-730. (doi:10.1038/nature03918)

Choi J, Shendrik I, Peacocke M, Peehl D, Buttyan R, Ikeguchi EF Katz AE \& Benson MC 2000 Expression of senescence-associated beta-galactosidase in enlarged prostates from men with benign prostatic hyperplasia. Urology 56 160-166. (doi:10.1016/S00904295(00)00538-0)

Coppe JP, Kauser K, Campisi J \& Beausejour CM 2006 Secretion of vascular endothelial growth factor by primary human fibroblasts at senescence. Journal of Biological Chemistry 281 29568-29574. (doi:10.1074/jbc.M603307200)

Coppe JP, Patil CK, Rodier F, Sun Y, Munoz DP, Goldstein J, Nelson PS, Desprez PY \& Campisi J 2008 Senescence-associated secretory phenotypes reveal cell-nonautonomous functions of oncogenic RAS and the p53 tumor suppressor. PLoS Biology 6 2853-2868.

Coppe JP, Desprez PY, Krtolica A \& Campisi J 2010 The senescenceassociated secretory phenotype: the dark side of tumor suppression. Annual Review of Pathology 5 99-118.(doi:10.1146/annurevpathol-121808-102144)

Crasta K, Ganem NJ, Dagher R, Lantermann AB, Ivanova EV, Pan Y, Nezi L, Protopopov A, Chowdhury D \& Pellman D 2012 DNA breaks and chromosome pulverization from errors in mitosis. Nature $\mathbf{4 8 2}$ 53-58. (doi:10.1038/nature10802)

Davalos AR, Coppe JP, Campisi J \& Desprez PY 2010 Senescent cells as a source of inflammatory factors for tumor progression. Cancer Metastasis Reviews 29 273-283. (doi:10.1007/s10555-010-9220-9)

Decordier I, Cundari E \& Kirsch-Volders M 2008 Survival of aneuploid, micronucleated and/or polyploid cells: crosstalk between ploidy control and apoptosis. Mutation Research 651 30-39. (doi:10.1016/j. mrgentox.2007.10.016)

Diaz-Martinez LA, Karamysheva ZN, Warrington R, Li B, Wei S, Xie XJ, Roth MG \& Yu H 2014 Genome-wide siRNA screen reveals coupling between mitotic apoptosis and adaptation. EMBO Journal 33 1960-1976. (doi:10.15252/embj.201487826)

Elhajouji A, Cunha M \& Kirsch-Volders M 1998 Spindle poisons can induce polyploidy by mitotic slippage and micronucleate mononucleates in the cytokinesis-block assay. Mutagenesis 13 193-198. (doi:10.1093/mutage/13.2.193)

Freund A, Patil CK \& Campisi J 2011 p38MAPK is a novel DNA damage response-independent regulator of the senescence-associated secretory phenotype. EMBO Journal 30 1536-1548. (doi:10.1038/ emboj.2011.69)

Fu Y, Ye D, Chen H, Lu W, Ye F \& Xie X 2007 Weakened spindle checkpoint with reduced BubR1 expression in paclitaxel-resistant ovarian carcinoma cell line SKOV3-TR30. Gynecologic Oncology 105 66-73. (doi:10.1016/j.ygyno.2006.10.061)

Fujiwara T, Bandi M, Nitta M, Ivanova EV, Bronson RT \& Pellman D 2005 Cytokinesis failure generating tetraploids promotes tumorigenesis in p53-null cells. Nature 437 1043-1047. (doi:10.1038/nature04217)
Gascoigne KE \& Taylor SS 2008 Cancer cells display profound intra- and interline variation following prolonged exposure to antimitotic drugs. Cancer Cell 14 111-122. (doi:10.1016/j.ccr.2008.07.002)

Habu T \& Matsumoto T 2013 p31(comet) inactivates the chemically induced Mad2-dependent spindle assembly checkpoint and leads to resistance to anti-mitotic drugs. Springerplus 2 562. (doi:10.1186/ 2193-1801-2-562)

Harley ME, Allan LA, Sanderson HS \& Clarke PR 2010 Phosphorylation of Mcl-1 by CDK1-cyclin B1 initiates its Cdc20-dependent destruction during mitotic arrest. EMBO Journal 29 2407-2420. (doi:10.1038/emboj.2010.112)

He Y, Yan D, Zheng D, Hu Z, Li H \& Li J 2016 Cell division cycle 6 promotes mitotic slippage and contributes to drug resistance in paclitaxel-treated cancer cells. PLOS ONE11 e0162633. (doi:10.1371/ journal.pone.0162633)

Herranz N, Gallage S, Mellone M, Wuestefeld T, Klotz S, Hanley CJ, Raguz S, Acosta JC, Innes AJ, Banito A, et al. 2015 mTOR regulates MAPKAPK2 translation to control the senescence-associated secretory phenotype. Nature Cell Biology 17 1205-1217. (doi:10.1038/ncb3225)

Huang HC, Shi J, Orth JD \& Mitchison TJ 2009 Evidence that mitotic exit is a better cancer therapeutic target than spindle assembly. Cancer Cell 16 347-358. (doi:10.1016/j.ccr.2009.08.020)

Huang HC, Mitchison TJ \& Shi J 2010 Stochastic competition between mechanistically independent slippage and death pathways determines cell fate during mitotic arrest. PLOS ONE 5 e15724. (doi:10.1371/journal.pone.0015724)

Jordan MA \& Wilson L 2004 Microtubules as a target for anticancer drugs. Nature Reviews Cancer 4 253-265. (doi:10.1038/nrc1317)

Krtolica A, Parrinello S, Lockett S, Desprez PY \& Campisi J 2001 Senescent fibroblasts promote epithelial cell growth and tumorigenesis: a link between cancer and aging. PNAS 98 12072-12077. (doi:10.1073/pnas.211053698)

Kuilman T \& Peeper DS 2009 Senescence-messaging secretome: SMS-ing cellular stress. Nature Reviews Cancer 9 81-94. (doi:10.1038/nrc2560)

Kuilman T, Michaloglou C, Vredeveld LC, Douma S, van Doorn R, Desmet CJ, Aarden LA, Mooi WJ \& Peeper DS 2008 Oncogeneinduced senescence relayed by an interleukin-dependent inflammatory network. Cell 133 1019-1031. (doi:10.1016/ j.cell.2008.03.039)

Laberge RM, Sun Y, Orjalo AV, Patil CK, Freund A, Zhou L, Curran SC, Davalos AR, Wilson-Edell KA, Liu S, et al. 2015 MTOR regulates the pro-tumorigenic senescence-associated secretory phenotype by promoting IL1A translation. Nature Cell Biology 17 1049-1061. (doi:10.1038/ncb3195)

Lara-Gonzalez P, Westhorpe FG \& Taylor SS 2012 The spindle assembly checkpoint. Current Biology 22 R966-R980. (doi:10.1016/j. cub.2012.10.006)

Ma HT, Chan YY, Chen X, On KF \& Poon RY 2012 Depletion of p31comet protein promotes sensitivity to antimitotic drugs. Journal of Biological Chemistry 287 21561-21569. (doi:10.1074/jbc. M112.364356)

Majumder PK, Grisanzio C, O'Connell F, Barry M, Brito JM, Xu Q Guney I, Berger R, Herman P, Bikoff R, et al. 2008 A prostatic intraepithelial neoplasia-dependent p27 Kip1 checkpoint induces senescence and inhibits cell proliferation and cancer progression. Cancer Cell 14 146-155. (doi:10.1016/j.ccr.2008.06.002)

Makarovskiy AN, Siryaporn E, Hixson DC \& Akerley W 2002 Survival of docetaxel-resistant prostate cancer cells in vitro depends on phenotype alterations and continuity of drug exposure. Cellular and Molecular Life Sciences 59 1198-1211. (doi:10.1007/ s00018-002-8498-3)

Martin SK, Pu H, Penticuff JC, Cao Z, Horbinski C \& Kyprianou N 2016 Multinucleation and mesenchymal-to-epithelial transition alleviate resistance to combined cabazitaxel and antiandrogen therapy in 
advanced prostate cancer. Cancer Research 76 912-926. (doi:10.1158/0008-5472.CAN-15-2078)

Michaloglou C, Vredeveld LC, Soengas MS, Denoyelle C, Kuilman T, van der Horst CM, Majoor DM, Shay JW, Mooi WJ \& Peeper DS 2005 BRAFE600-associated senescence-like cell cycle arrest of human naevi. Nature 436 720-724. (doi:10.1038/nature03890)

Mittal K, Donthamsetty S, Kaur R, Yang C, Gupta MV, Reid MD, Choi DH, Rida PCG \& Aneja R 2017 Multinucleated polyploidy drives resistance to Docetaxel chemotherapy in prostate cancer. British Journal of Cancer 116 1186-1194. (doi:10.1038/bjc.2017.78)

Mosieniak G \& Sikora E 2010 Polyploidy: the link between senescence and cancer. Current Pharmaceutical Design 16 734-740. (doi:10.2174/ 138161210790883714

Musacchio A \& Ciliberto A 2012 The spindle-assembly checkpoint and the beauty of self-destruction. Nature Structural and Molecular Biology 19 1059-1061. (doi:10.1038/nsmb.2429)

Nardella C, Clohessy JG, Alimonti A \& Pandolfi PP 2011 Pro-senescence therapy for cancer treatment. Nature Reviews Cancer 11 503-511. (doi:10.1038/nrc3057)

Ohashi A, Ohori M, Iwai K, Nakayama Y, Nambu T, Morishita D, Kawamoto T, Miyamoto M, Hirayama T, Okaniwa M, et al. 2015 Aneuploidy generates proteotoxic stress and DNA damage concurrently with p53-mediated post-mitotic apoptosis in SAC-impaired cells. Nature Communications 6 7668. (doi:10.1038/ncomms8668)

Orjalo AV, Bhaumik D, Gengler BK, Scott GK \& Campisi J 2009 Cell surface-bound IL-1alpha is an upstream regulator of the senescenceassociated IL-6/IL-8 cytokine network. PNAS 106 17031-17036. (doi:10.1073/pnas.0905299106)

Orth JD, Loewer A, Lahav G \& Mitchison TJ 2012 Prolonged mitotic arrest triggers partial activation of apoptosis, resulting in DNA damage and p53 induction. Molecular Biology of the Cell 23 567-576. (doi:10.1091/mbc.E11-09-0781)

Panvichian R, Orth K, Day ML, Day KC, Pilat MJ \& Pienta KJ 1998 Paclitaxel-associated multimininucleation is permitted by the inhibition of caspase activation: a potential early step in drug resistance. Cancer Research 58 4667-4672.

Parrinello S, Coppe JP, Krtolica A \& Campisi J 2005 Stromal-epithelial interactions in aging and cancer: senescent fibroblasts alter epithelial cell differentiation. Journal of Cell Science 118 485-496. (doi:10.1242/ jcs.01635)

Prencipe M, Fitzpatrick P, Gorman S, Tosetto M, Klinger R, Furlong F, Harrison M, O'Connor D, Roninson IB, O'Sullivan J, et al. 2009 Cellular senescence induced by aberrant MAD2 levels impacts on paclitaxel responsiveness in vitro. British Journal of Cancer 101 1900-1908. (doi:10.1038/sj.bjc.6605419)

Quignon F, Rozier L, Lachages AM, Bieth A, Simili M \& Debatisse M 2007 Sustained mitotic block elicits DNA breaks: one-step alteration of ploidy and chromosome integrity in mammalian cells. Oncogene 26 165-172. (doi:10.1038/sj.onc.1209787)

Rieder CL \& Maiato H 2004 Stuck in division or passing through: what happens when cells cannot satisfy the spindle assembly checkpoint. Developmental Cell 7 637-651. (doi:10.1016/j.devcel.2004.09.002)

Roberson RS, Kussick SJ, Vallieres E, Chen SY \& Wu DY 2005 Escape from therapy-induced accelerated cellular senescence in p53-null lung cancer cells and in human lung cancers. Cancer Research 65 2795-2803. (doi:10.1158/0008-5472.CAN-04-1270)

Roberts JR, Allison DC, Donehower RC \& Rowinsky EK 1990 Development of polyploidization in taxol-resistant human leukaemia cells in vitro. Cancer Research 50 710-716.

Ruhland MK, Loza AJ, Capietto AH, Luo X, Knolhoff BL, Flanagan KC, Belt BA, Alspach E, Leahy K, Luo J, et al. 2016 Stromal senescence establishes an immunosuppressive microenvironment that drives tumorigenesis. Nature Communications 7 11762. (doi:10.1038/ ncomms11762)

Sakurikar N, Eichhorn JM \& Chambers TC 2012 Cyclin-dependent kinase-1 (Cdk1)/cyclin B1 dictates cell fate after mitotic arrest via phosphoregulation of antiapoptotic Bcl-2 proteins. Journal of Biological Chemistry 287 39193-39204. (doi:10.1074/jbc. M112.391854)

Sakurikar N, Eichhorn JM, Alford SE \& Chambers TC 2014 Identification of a mitotic death signature in cancer cell lines. Cancer Letters $\mathbf{3 4 3}$ 232-238. (doi:10.1016/j.canlet.2013.09.036)

Shi J, Orth JD \& Mitchison T 2008 Cell type variation in responses to antimitotic drugs that target microtubules and kinesin-5. Cancer Research 68 3269-3276. (doi:10.1158/0008-5472.CAN-07-6699)

Shi J, Zhou Y, Huang HC \& Mitchison TJ 2011 Navitoclax (ABT-263) accelerates apoptosis during drug-induced mitotic arrest by antagonizing Bcl-xL. Cancer Research 71 4518-4526. (doi:10.1158/0008-5472.CAN-10-4336)

Sloss O, Topham C, Diez M \& Taylor S 2016 Mcl-1 dynamics influence mitotic slippage and death in mitosis. Oncotarget 7 5176-5192.

Sudo T, Nitta M, Saya H \& Ueno NT 2004 Dependence of paclitaxel sensitivity on a functional spindle assembly checkpoint. Cancer Research 64 2502-2508. (doi:10.1158/0008-5472.CAN-03-2013)

Thorpe PH, Gonzalez-Barrera S \& Rothstein R 2007 More is not always better: the genetic constraints of polyploidy. Trends in Genetics $\mathbf{2 3}$ 263-266. (doi:10.1016/j.tig.2007.03.016)

Toso A, Revandkar A, Di Mitri D, Guccini I, Proietti M, Sarti M, Pinton S, Zhang J, Kalathur M, Civenni G, et al. 2014 Enhancing chemotherapy efficacy in Pten-deficient prostate tumors by activating the senescence-associated antitumor immunity. Cell Reports 9 75-89. (doi:10.1016/j.celrep.2014.08.044)

Ventura A, Kirsch DG, McLaughlin ME, Tuveson DA, Grimm J, Lintault L, Newman J, Reczek EE, Weissleder R \& Jacks T 2007 Restoration of p53 function leads to tumour regression in vivo. Nature 445 661-665. (doi:10.1038/nature05541)

Vitale I, Galluzzi L, Senovilla L, Criollo A, Jemaa M, Castedo M \& Kroemer G 2011 Illicit survival of cancer cells during polyploidization and depolyploidization. Cell Death and Differentiation 18 1403-1413. (doi:10.1038/cdd.2010.145)

Wang Q, Wu PC, Dong DZ, Ivanova I, Chu E, Zeliadt S, Vesselle H \& Wu DY 2013 Polyploidy road to therapy-induced cellular senescence and escape. International Journal of Cancer 132 1505-1515. (doi:10.1002/ijc.27810)

Weaver BA \& Cleveland DW 2005 Decoding the links between mitosis, cancer, and chemotherapy: The mitotic checkpoint, adaptation, and cell death. Cancer Cell 8 7-12. (doi:10.1016/j.ccr.2005.06.011)

Xue W, Zender L, Miething C, Dickins RA, Hernando E, Krizhanovsky V, Cordon-Cardo C \& Lowe SW 2007 Senescence and tumour clearance is triggered by p53 restoration in murine liver carcinomas. Nature 445 656-660. (doi:10.1038/nature05529)

Yamada HY \& Gorbsky GJ 2006 Spindle checkpoint function and cellular sensitivity to antimitotic drugs. Molecular Cancer Therapy 5 2963-2969. (doi:10.1158/1535-7163.MCT-06-0319)

Zhang S, Mercado-Uribe I, Xing Z, Sun B, Kuang J \& Liu J 2014 Generation of cancer stem-like cells through the formation of polyploid giant cancer cells. Oncogene 33 116-128. (doi:10.1038/ onc.2013.96)

Zhu Y, Zhou Y \& Shi J 2014 Post-slippage multinucleation renders cytotoxic variation in anti-mitotic drugs that target the microtubules or mitotic spindle. Cell Cycle 13 1756-1764. (doi:10.4161/cc.28672)

Received in final form 4 July 2017

Accepted 6 July 2017

Accepted preprint published online 6 July 2017 http://erc.endocrinology-journals.org

DOI: 10.1530/ERC-17-0147
C 2017 Society for Endocrinology Printed in Great Britain 